EPJ Web of Conferences 41, 11008 (2013)

DOI: $10.1051 /$ epjconf/20134111008

(C) Owned by the authors, published by EDP Sciences, 2013

\title{
Generation of trains of arbitrary waveforms and their repeated revivals
}

\author{
Masayuki Katsuragawa, Takayuki Suzuki, and Kanaka Raju Pandiri \\ Department of Engineering Science, University of Electro-Communications, 1-5-1 Chofugaoka, \\ Chofu-shi, Tokyo, Japan
}

\begin{abstract}
We report on manipulation and characterization of highly-discrete coherent spectrum. It is shown that trains of arbitrary optical waveforms are generated and furthermore they revive repeatedly by adding material positive dispersions.
\end{abstract}

\section{Introduction}

The generation and measurement of arbitrary optical waveforms (AOW) have had a significant impact in relation to the coherent control of chemical reactions, and optical communications. Lineby-line control, in which spectral lines are resolved and manipulated individually, leads to a fundamentally new regime for AOW generation [1]. Adiabatically generated Raman sidebands (Raman comb) [2-5] is suitable source for implementing line-by-line control since they have discrete characteristics typically a greater than $\mathrm{THz}$ frequency spacing and good mutual coherence. By using such Raman sidebands, a synthesized AOW can have an ultrahigh repetition rate corresponding to the wide frequency spacing of the Raman sidebands [5, 6]. Here, we report the synthesis of $10-\mathrm{THz}$ ultrahigh repetition rate AOW using rotational Raman sidebands $(J=2 \leftarrow 0)$ in parahydrogen [6]. We show that trains of rectangular and triangular pulses are stably produced, such as an ultrafast function generator. Furthermore, we also report another feature of the waveforms constructed with such highly-discrete coherent spectrum; that is, the arbitrary waveforms can be realized repeatedly by adding a material positive dispersion [7].

\section{Experimental and Results}

The entire system (Fig. 1) consists of three parts that generate Raman sidebands, manipulate their spectral phases, and measure the spectral phases to produce the obtained waveform together with an error signal for feedback.

Figure 2 shows the results when the target waveform was set as a train of rectangular pulses (gray dotted line in Fig. 2; duty ratio: 50\%). The blue curve in Fig. 2 is the corresponding effective target waveform in the time domain. We employed the process for converging the sidebands with the effective target. The obtained waveform (bold gray line) precisely matched the effective target (thin solid line). Note that this waveform was reduced for measuring the spectral phases of the sidebands with nanosecond pulsed envelopes, and they showed fine stability $(< \pm 0.1$ radians $)$ for several hours. 


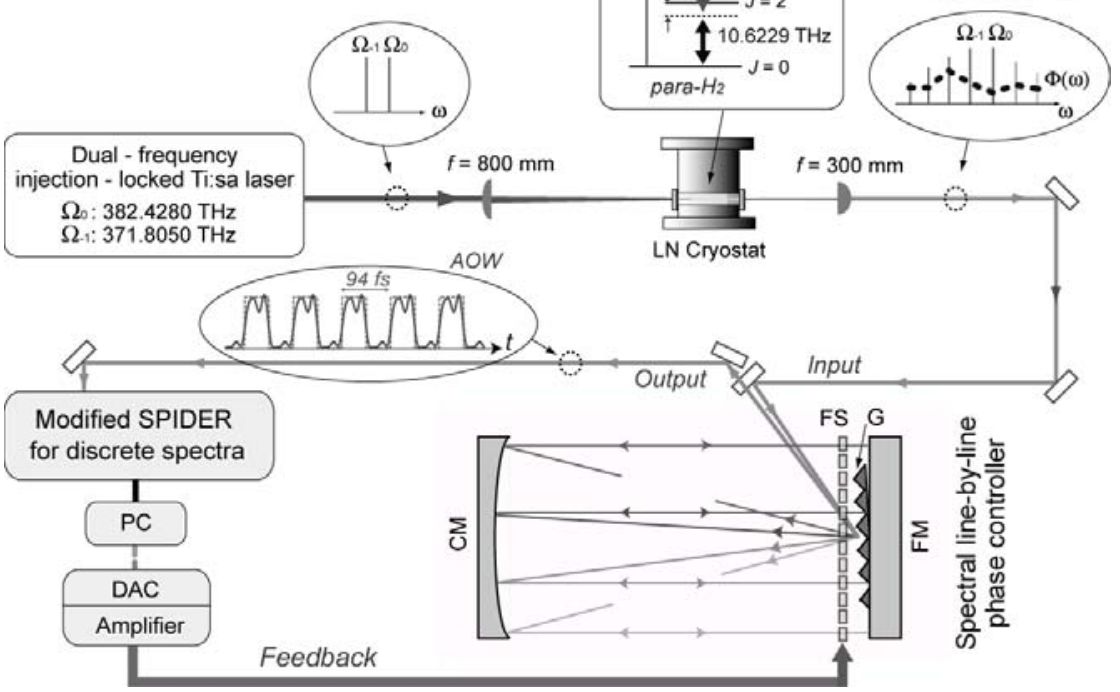

Fig. 1. Experimental setup. LN Cryostat, liquid nitrogen cryostat; G, grating; FS, fused silica plates; FM, folding mirror; CM, concave mirror; PC, personal computer; DAC, digital-analog converter; AOW, arbitrary optical waveform.

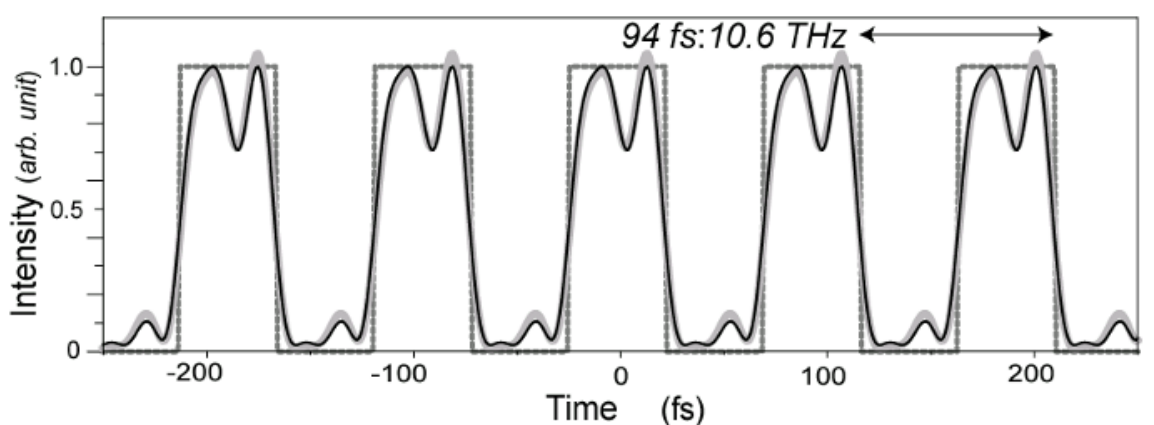

Fig. 2. Generation of an ultrahigh repetition rate train of rectangular pulses.

This shows that the ultrahigh repetition rate $(10.6229 \mathrm{THz})$ train of the rectangular waveform was stably produced with more than 10,000 successive pulses.

Another interesting feature on the optical waveforms consisting of such highly-discrete coherent spectrum is that we can revive a pulse shape repeatedly by adding a material positive dispersion [9]. We numerically and experimentally demonstrate the efficacy of this idea. Figure 3 show spectral phases for the Raman sidebands when the inserted thicknesses of a fused-silica prism pair (the dispersion controller: see the inset of Fig. 3) in the optical path was changed every $5 \mathrm{~mm}$ from 10 to $40 \mathrm{~mm}$. Solid, dashed and shaded curves respectively indicate initial, FTL, and observed spectral phases. The two indicated FTL conditions (dashed) are well realized repeatedly. 


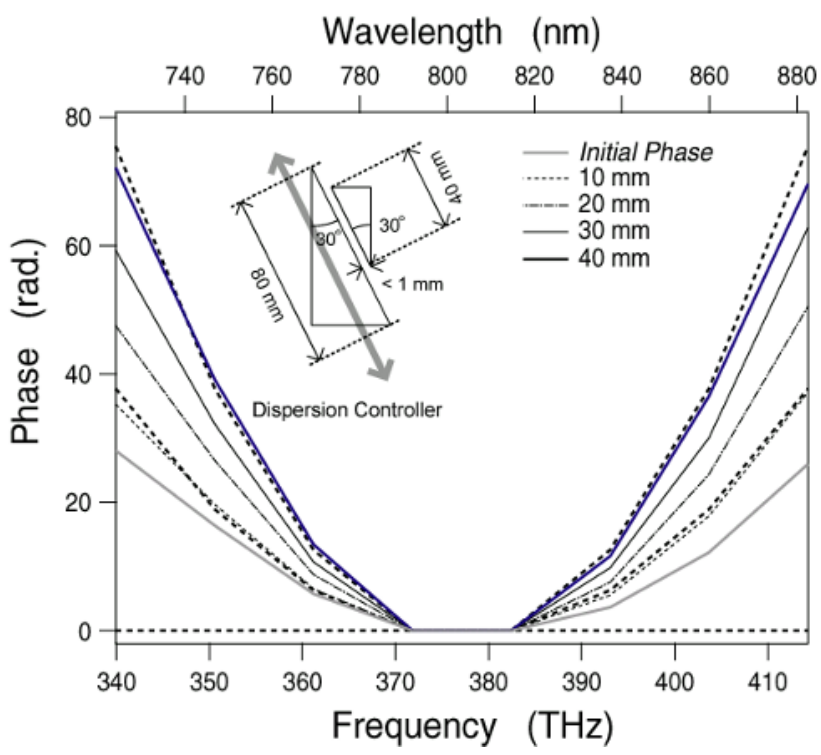

Fig. 3. Observed spectral phases for various thicknesses of the insertion material.

\section{Summary}

We have shown that trains of arbitrary optical waveforms were succesfuuly generated with an ultrahiugh repetition rate of $10 \mathrm{THz}$ such as 'an ultrahigh frequency function generator' by manipulating a highly-discrete coherent spectrum produced thorough adiabatic excitation of the near-maximal Raman coherence in gaseous molecular parahydrogen. It is also shown that such trains of arbitrary optical waveforms can be revival repeatedly by simply adding material positive dispersions.

\section{References}

1. Z. Jiang, C.-B. Huang, D. E. Leaird, and A. M. Weiner, Nat. Photonics 1, 463 (2007).

2. S. E. Harris and A. V. Sokolov, Phys. Rev. A 55, R4019 (1997).

3. A. V. Sokolov, D. R. Walker, D. D. Yavuz, G. Y. Yin, and S. E. Harris, Phys. Rev. Lett. 85, 562 (2000).

4. J.Q. Liang, M. Katsuragawa, F. Le Kien, and K. Hakuta, Phys. Rev. Lett. 85, 2474 (2000).

5. Chan, H. -S. et al., Science 331, 1165 (2011).

6. K. R. Pandiri and M. Katsuragawa, New Journal of Physics 13, 023030 (2011).

7. T. Suzuki and M. Katsuragawa, Opt. Express 18, 23088 (2010).

8. T. Suzuki, N. Sawayama, and M. Katsuragawa, Opt. Lett. 33, 2809 (2008).

9. N. K. Berger, B. Levit, A. Bekker, and B. Fischer, IEEE Photon. Technol. Lett. 16(8), 1855 (2004). 